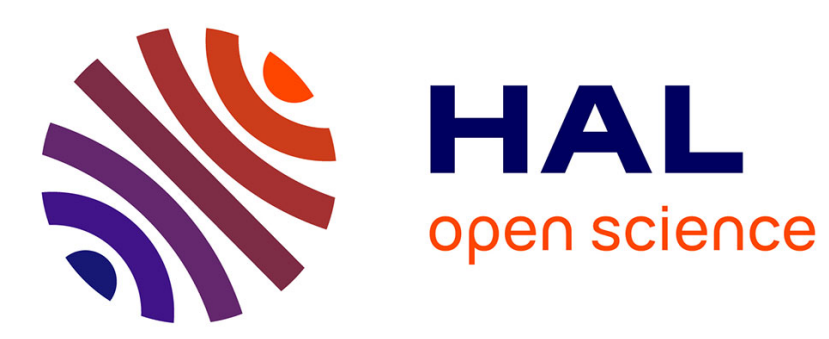

\title{
Élaboration et transformation d'alliages binaires à base de niobium à l'état de grande pureté
}

\author{
R. Geschier, A. Clauss, H. Forestier, J.P. Givord, M. Armand
}

\section{To cite this version:}

R. Geschier, A. Clauss, H. Forestier, J.P. Givord, M. Armand. Élaboration et transformation d'alliages binaires à base de niobium à l'état de grande pureté. Revue de Physique Appliquée, 1970, 5 (3), pp.443-447. 10.1051/rphysap:0197000503044300 . jpa-00243414

\section{HAL Id: jpa-00243414 https://hal.science/jpa-00243414}

Submitted on 1 Jan 1970

HAL is a multi-disciplinary open access archive for the deposit and dissemination of scientific research documents, whether they are published or not. The documents may come from teaching and research institutions in France or abroad, or from public or private research centers.
L'archive ouverte pluridisciplinaire HAL, est destinée au dépôt et à la diffusion de documents scientifiques de niveau recherche, publiés ou non, émanant des établissements d'enseignement et de recherche français ou étrangers, des laboratoires publics ou privés. 


\title{
ÉLABORATION ET TRANSFORMATION D'ALLIAGES BINAIRES A BASE DE NIOBIUM A L'ÉTAT DE GRANDE PURETÉ
}

\author{
par R. GESCHIER, A. CLAUSS et H. FORESTIER \\ Département Sciences de Matériaux, E. N. S. C., Strasbourg, 1, rue Blaise-Pascal, 67, Strasbourg \\ J. P. GIVORD et M. ARMAND \\ Laboratoire de Recherches de Venthon, Sté Ugine-Kuhlmann, 73, Albertville
}

\begin{abstract}
Résumé. - A partir de métaux très purs les alliages binaires suivants ont été élaborés par fusion par bombardement électroniques : $\mathrm{Nb}-\mathrm{Zr}$ à $2,0,3,85,5,45 \%$ at $\mathrm{Zr}$ et $\mathrm{Nb}-\mathrm{Mo}$ à $5,3 \%$ at Mo. Un alliage $\mathrm{Nb}-\mathrm{Ti}$ à $4,65 \%$ at $\mathrm{Ti}$ a été préparé au four à arc. Ces alliages, sous forme de lingots de $4 \mathrm{~kg}$ environ, présentent une bonne homogénéité. Leur pureté est supérieure à $99,97 \%$.

Après forgeage en plats, ces alliages sont transformés à froid, soit par laminage jusqu'à $1 \mathrm{~mm}$, soit par retreinte à l'état de fils jusqu'à $0,3 \mathrm{~mm}$ de diamètre. Les conditions opératoires sont précisées et on rend compte des difficultés rencontrées et de l'expérience acquise.
\end{abstract}

\begin{abstract}
This paper concerns the preparation and the transformation of $\mathrm{Nb}$-rich alloys made by melting high purity metals. $\mathrm{Nb}-\mathrm{Zr}$ and $\mathrm{Nb}-\mathrm{Mo}$ alloys have been prepared by electronbeam melting; $\mathrm{Nb}-\mathrm{Ti}$ alloy has been prepared by vacuum-arc melting.

After cold-forging of the cast ingots, the alloys have been either cold-rolled until $1 \mathrm{~mm}$ thick, or cold-swaged until $0,3 \mathrm{~mm}$ diameter. Experimental conditions are precised.
\end{abstract}

Comparé aux autres métaux réfractaires, tantale, molybdène et tungstène, le niobium offre l'avantage de réunir un ensemble unique de caractéristiques et propriétés : température de fusion élevée, faible section de capture vis-à-vis des neutrons, conductibilité et coefficient de dilatation thermiques élevés, température de transition supraconductrice élevée, etc.

La température de transition ductile-fragile du niobium et du tantale purs est très inférieure à l'ambiante (environ $-200^{\circ} \mathrm{C}$ ), contrairement à celle du molybdène et du tungstène. Enfin, la densité relativement faible du niobium confère à ce métal un net avantage tant sur le plan économique que du point de vue de son utilisation comme élément de structure.

Cette combinaison intéressante de propriétés a suscité de nombreuses recherches dans des domaines d'application très divers : génie nucléaire, génie chimique, supraconductivité, éléments de structure à haute température etc. En outre, la découverte d'importants gisements de minerais de niobium, au Canada et au Brésil, vers 1950, a entraîné un développement accéléré de ce métal.

Parallèlement, des alliages à base de niobium ont été rapidement développés. L'addition d'éléments alliants avait principalement pour but d'augmenter les caractéristiques mécaniques, de conserver une bonne malléabilité et d'accroître la résistance à l'oxyda- tion à température élevée. Mais il est apparu très rapidement qu'il n'existait pas un alliage supérieur à tous les autres : l'optimalisation d'une caractéristique est généralement obtenue aux dépens de l'une ou des deux autres caractéristiques. De plus, le développement de ces alliages complexes s'est effectué le plus souvent de façon empirique, sans connaissance approfondie des systèmes binaires et en négligeant la présence d'impuretés non métalliques. L'étude de l'inflence de ces impuretés sur le comportement des alliages à base de niobium se borne souvent à des observations sur quelques alliages à teneurs en impuretés non contrôlées.

Dans le cadre d'un contrat D. G. R. S. T., nous avons entrepris un travail qui devrait nous permettre de combler en partie cette lacune. L'objectif de notre étude est de préciser l'influence spécifique de certaines impuretés non métalliques, $\mathrm{O}, \mathrm{N}$ et $\mathrm{C}$, sur les propriétés de quelques alliages binaires comportant l'un des éléments préconisés dans les formules actuellement les plus intéressantes.

Au départ se posait le problème de la contamination contrôlée des alliages. Deux voies s'offraient à nous : introduire l'impureté dans l'alliage au moment de son élaboration par fusion, ou bien introduire l'impureté ultérieurement, par diffusion à haute température, dans l'alliage très pur. Nous avons choisi cette deuxième voie qui présente sur la première un certain 
nombre d'avantages : elle facilite la transformation des alliages, limite la contamination et permet d'obtenir des teneurs en impuretés très précises dans une large gamme de concentration au fur et à mesure de l'avancement de l'étude.

La première étape, fondamentale, de notre étude consistait donc à préparer des alliages à un très grand degré de pureté. Les alliages binaires retenus dans le cadre du contrat sont :

$\mathrm{Nb}-1 \%, 3 \%$ et $5 \%$ at. $\mathrm{Zr}$.

$\mathrm{Nb}-5 \%$ at. Mo.

$\mathrm{Nb}-5 \%$ at. $\mathrm{Ti}$.

Nous exposons dans ce mémoire les techniques d'élaboration et de transformation de ces cinq alliages, ainsi que les données analytiques qui leur correspondent.

I. Elaboration des alliages. - A. MATÉRIAUX DE DÉPART. - Le niobium ultra-pur utilisé a été élaboré par la Société Ugine-Kuhlmann. Le procédé de fabrication comporte la purification chimique par distillation des chlorures, la transformation du chlorure $\mathrm{NbCl}_{5}$ en oxyde $\mathrm{Nb}_{2} \mathrm{O}_{5}$ et la carboréduction sous vide de cet oxyde en niobium métallique. La purification finale est réalisée au four à bombardement électronique [1].

Les éléments d'alliages sont d'une part du zirconium et du titane Van Arkel, d'autre part du molybdène purifié par bombardement électronique.

Les teneurs en impuretés de ces différents métaux sont indiquées dans le tableau I.

B. Choix DE LA TeChNique DE FUSION. - Les principales techniques de consolidation utilisées dans le cas des métaux et alliages réfractaires sont le frittage, la fusion à l'arc et la fusion par bombardement électronique. Il peut être intéressant, en outre, de combiner deux de ces procédés.

Ainsi, une structure à grains fins peut être obtenue par frittage d'une poudre provenant d'un alliage préalablement fondu par bombardement électronique. De même, l'addition d'un élément volatil à un alliage élaboré par bombardement électronique peut se faire au cours d'une fusion finale à l'arc.

Le réglage très souple de la vitesse de fusion, la possibilité de maintenir le bain en fusion sur une certaine profondeur et le vide élevé régnant dans l'enceinte, font du bombardement électronique non seulement une technique de fusion intéressante mais également un excellent procédé de purification, tant en impuretés métalliques qu'en impuretés non métalliques [1] [2].

Cependant, l'évaporation d'un élément plus volatil que le métal de base, intéressante lors de la purification, peut rendre impossible l'élaboration par bombardement électronique d'un alliage devant comporter cet élément. Il en est ainsi de l'alliage $\mathrm{Nb}$-Ti qui, par suite de la forte tension de vapeur du titane, a été élaboré au four à arc.

Le zirconium et le molybdène, plus volatils que le niobium, ont également tendance à s'évaporer. Il est néanmoins possible d'adapter les conditions de fusion par bombardement électronique de façon à contrôler les pertes. Les alliages $\mathrm{Nb}-\mathrm{Zr}$ et $\mathrm{Nb}-\mathrm{Mo}$ ont été élaborés par cette technique. En l'absence de données permettant d'évaluer ces pertes, celles-ci ont été estimées expérimentalement par fusion d'un lingot d'essai.

C. Fusion des Alliages. - 1. Alliages $\mathrm{Nb}-\mathrm{Zr}$ et Nb-Mo. - Le four utilisé est un four à faisceaux d'électrons Heraeus d'une puissance de $150 \mathrm{~kW}$. Il comporte trois canons à électrons. La puissance utilisée lors des fusions est de $23 \mathrm{~kW}$ par canon; le vide moyen régnant dans l'enceinte est de $3 \times 10^{-5}$ torr.

La lingotière consiste en un creuset en cuivre de $60 \mathrm{~mm}$ de diamètre. Le fond du creuset est constitué par un cylindre de niobium assujetti à une tige d'entraînement. Deux boutons de la nuance de l'alliage considéré sont coulés sur cette base et forment l'amorce $\mathrm{du}$ lingot. Chaque alliage subit deux fusions.

a. Première fusion. - La première fusion est effectuée sur un barreau de copeaux comprimés à la presse. L'élément d'addition est réparti manuellement au sein de ce barreau, de la façon la plus homogène possible. Ce barreau de dimensions $50 \times 50 \times 500 \mathrm{~mm}$ et d'un poids approximatif de $5,5 \mathrm{~kg}$, est suspendu dans le four à l'aide d'un fil de niobium ultra-pur.

La descente $\mathrm{du}$ barreau est régulière; elle est calculée de manière à obtenir une vitesse de fusion de $40 \mathrm{~kg} / \mathrm{h}$ environ. Cette vitesse relativement élevée

\section{TABLEAU 1}

Analyse des impuretés du Niobium et des éléments d'alliage

\begin{tabular}{|c|c|c|c|c|c|c|c|}
\hline & $\mathrm{O}_{\mathrm{ppm}}$ & $\mathrm{N}_{\mathrm{ppm}}$ & $\mathrm{C}_{\mathrm{ppm}}$ & $\mathrm{H}_{\mathrm{ppm}}$ & $\begin{array}{c}\Sigma(\mathrm{O}+\underset{\mathrm{ppm}}{\mathrm{N}}+\mathrm{C}+\mathrm{H}) \\
-\end{array}$ & $\begin{array}{c}\Sigma \text { impuretés } \\
\text { métalliques } \\
\text { ppm }\end{array}$ & Pureté \\
\hline $\mathrm{Nb}$ & $<10$ & 25 & 23 & $<1$ & 60 & $<90$ & $99,985 \%$ \\
\hline $\mathrm{Zr}$ & 24 & 5 & 31 & 11 & 70 & $<450$ & $99,95 \%$ \\
\hline Mo & 8 & 10 & 12 & - & 30 & 150 & $99,98 \%$ \\
\hline $\mathrm{Ti}$ & 20 & 20 & 10 & - & 50 & 750 & $99,92 \%$ \\
\hline
\end{tabular}


TABLEAU II

Analyse des éléments d'alliage

\begin{tabular}{|c|c|c|c|c|c|}
\hline Elément d'alliage & & $\mathrm{Zr} \%$ poids & & $\begin{array}{c}\text { Mo \% en } \\
\text { poids }\end{array}$ & $\begin{array}{l}\mathrm{Ti} \% \text { en } \\
\text { poids }\end{array}$ \\
\hline Composition avant fusion & 2,2 & 4,0 & 6,1 & $\overline{5,3}$ & 2,57 \\
\hline $\begin{array}{l}\text { Tête } \ldots \ldots \\
\text { Pied } \ldots\end{array}$ & $\begin{array}{l}2,00 \\
2,02\end{array}$ & $\begin{array}{l}3,90 \\
3,80\end{array}$ & $\begin{array}{l}5,30 \\
5,55\end{array}$ & $\begin{array}{l}4,70^{*} \\
4,45^{*}\end{array}$ & $\begin{array}{l}2,45 \\
3,05^{*}\end{array}$ \\
\hline Composition moyenne & $1,99 \pm 0,10$ & $3,83 \pm 0,10$ & $5,4 \pm 0,20$ & $5,45 \pm 0,20$ & $2,45 \pm 0,10$ \\
\hline Composition moyenne en $\%$ at. & 2,00 & 3,85 & 5,45 & 5,3 & 4.65 \\
\hline
\end{tabular}

$\left(^{*}\right)$ Ces valeurs d'analyses correspondent à des zones hétérogènes limitées aux extrémités des lingots $(20 \mathrm{~mm}$ dans le cas de $\mathrm{Nb}-\mathrm{Mo}, 5 \mathrm{~mm}$ dans le cas de $\mathrm{Nb}$-Ti). La composition moyenne correspond à la partie homogène de ces lingots.

permet de limiter les pertes par volatilisation au cours de cette fusion de mise en forme.

b. Seconde fusion. - Le lingot de première fusion est scié en deux moitiés dans le sens longitudinal. Ces deux moitiés sont placées tête-bêche dans le creuset et soudées, dans le four, à un petit barreau de niobium ultra-pur assujetti préalablement à la tête d'électrode.

Au cours de cette seconde fusion la descente de l'électrode n'est pas continue. Elle est interrompue périodiquement de façon programmée; la vitesse moyenne de fusion est de $25 \mathrm{~kg} / \mathrm{h}$.

Le choix des deux vitesses de fusion est important car les pertes par évaporation et l'homogénéité des lingots en dépendent. En particulier, le mouvement de l'électrode doit être calculé de manière à assurer un maintien à l'état liquide suffisant sans surchauffe importante du bain.

2. Alliage $\mathrm{Nb}-\mathrm{Ti}$. - Les copeaux de niobium et de titane ont été comprimés en huit barreaux de dimensions $25 \times 25 \times 250 \mathrm{~mm}$. Ces barreaux ont été soudés bout à bout en deux électrodes de un mètre de long.

Lors de la soudure de la tête d'électrode en niobium ultra-pur sur le premier barreau, soudure réalisée directement dans le four à arc, nous avons constaté un bleuissement assez important du barreau. Afin d'éliminer cette cause de contamination, les barreaux ont subi un traitement de dégazage sous vide à $800^{\circ} \mathrm{C}$ : le vide est cassé à l'argon et les barreaux, conservés sous argon, ont été soudés en boîte à gants sous argon purifié.

Les deux électrodes ont été fondues au four à arc en deux lingots de première fusion de $50 \mathrm{~mm}$ de diamètre. Après écroutage, ces deux lingots ont été refondus en un lingot de $60 \mathrm{~mm}$ de diamètre.

II. Analyses. - Les lingots bruts de fusion subissent un écroutage de 1 à $2 \mathrm{~mm}$ suivant leur état de surface. De plus, des tranches de $1 \mathrm{~cm}$ environ sont éliminées aux extrémités des lingots. Leurs dimensions finales sont: longueur $20 \mathrm{~cm}$, diamètre $56 \mathrm{~mm}$ environ. Les prélèvements pour analyse sont effectués en tête et pied ainsi qu'aux tiers et deux tiers de chaque lingot. D'autres analyses ont été effectuées ultérieure- ment sur les produits transformés. L'ensemble des résultats est résumé ci-après.

1. ElÉMENTS D'Alliage. - Les éléments d'alliage ont été dosés par fluorescence $X$. Nous avons déterminé les conditions de dosage de chaque élément et tracé les courbes d'étalonnage ; dans le domaine de concentrations envisagé ( 0 à $5 \%$ at.) ce sont des droites.

Les résultats de ces analyses sont indiqués dans le tableau II. De l'examen de ce tableau ressortent les points suivants :

- les pertes en zirconium ont été nettement inférieures aux estimations préliminaires; elles sont de l'ordre de $10 \%$ de la teneur initiale ;

- le lingot Nb-Mo est appauvri en tête et pied, ce qui rend impossible l'évaluation des pertes en molybdène ;

- le lingot $\mathrm{Nb}-\mathrm{Ti}$ est enrichi en pied.

Les analyses complémentaires, effectuées sur les produits en cours de transformation, ont confirmé la bonne homogénéité des alliages $\mathrm{Nb}-\mathrm{Zr}$. Dans le cas des alliages $\mathrm{Nb}-\mathrm{Mo}$ et $\mathrm{Nb}-\mathrm{Ti}$, des analyses sur tôles ont montré que l'hétérogénéité en molybdène et titane ne concernait qu'une zone limitée aux extrémités du lingot. Cette hétérogénéité résulte probablement d'une mauvaise répartition de l'élément alliant dans le barreau comprimé initial. La préparation d'un barreau homogène est en effet délicate étant donnée la diversité de forme et de poids des copeaux.

2. IMPURETÉS NON MÉTALLIQUES. - L'oxygène et l'hydrogène sont dosés par fusion réductrice sous vide, l'azote par micro kjeldahl, le carbone par combustion et conductimétrie différentielle. Les résultats de ces analyses sont donnés dans le tableau III.

\section{TABleau III}

\section{Analyse des impuretés non métalliques}

\begin{tabular}{|c|c|c|c|c|c|}
\hline \multirow[b]{2}{*}{$\mathrm{Nb}-2 \mathrm{Zr} \ldots}$. & \multicolumn{2}{|c|}{$\mathrm{O}_{\mathrm{ppm}}$} & \multirow{2}{*}{$20 \frac{N_{\text {ppm }}}{\grave{a ̀}} 35$} & \multirow{2}{*}{$\frac{\mathrm{C}_{\mathrm{ppm}}}{15 \overline{\text { à }} 25}$} & \multirow{2}{*}{$\frac{\mathrm{H}_{\mathrm{ppm}}}{2}$} \\
\hline & 15 à & 35 & & & \\
\hline $\mathrm{Nb}-3,85 \mathrm{Zr} .$. & 20 à & 35 & 20 à 50 & 15 à 25 & \\
\hline $\mathrm{Nb}-5,4 \mathrm{Zr}$ & 15 à & 25 & 15 à 30 & 10 à 20 & 2 \\
\hline Nb-5,3 Mo .. & 25 à & 45 & 40 à 60 & 15 à 20 & \\
\hline $\mathrm{Nb}-2,45 \mathrm{Ti} \ldots$ & 70 à & 10 & 50 à 80 & 25 à 35 & 2 \\
\hline
\end{tabular}


TABleau IV Analyse type des impuretés métalliques d'un alliage $\mathrm{Nb}-\mathrm{Zr}$

$\begin{array}{lccccccccc}\text { Impuretés } \ldots . . . . . & \text { Ta } & \text { W } & \text { Mo } & \text { Ti } & \text { Si } & \text { Ni } & \text { Sn } & \text { Al } & \frac{\mathrm{Fe}}{-} \\ \text { Teneur en ppm ..... } & \frac{-}{23} & <20 & <40 & <20 & 11 & <10 & <20 & <50 & 40\end{array}$

D'après ce tableau, la teneur en oxygène des alliages est sensiblement plus élevée que dans le niobium de départ. La teneur totale en éléments non métalliques reste faible pour les alliages $\mathrm{Nb}-\mathrm{Zr}$, soit $80 \mathrm{ppm}$ environ. Par contre, l'alliage $\mathrm{Nb}-\mathrm{Ti}$ a une teneur totale sensiblement plus élevée (environ $200 \mathrm{ppm}$ ).

La contamination par l'oxygène intervient probablement lors de la mise en copeaux qui, malgré les précautions prises, entraîne une légère pollution du métal. Dans le cas de l'alliage $\mathrm{Nb}-\mathrm{Ti}$, le procédé de fusion augmente encore cette contamination qui peut cependant être considérée comme limitée.

3. IMPURETÉS MÉTALLIQUeS. - Les teneurs en impuretés métalliques sont déterminées par fluorescence $\mathrm{X}$ (W, Ta, Mo, Ti, Zr) et par spectrographie d'arc [1] [3].

A l'exception du fer, dont la teneur augmente de $30 \mathrm{ppm}$ environ, les teneurs en impuretés métalliques sont identiques à celles du niobium de départ.

Le tableau IV présente une analyse type des principales impuretés métalliques d'un alliage $\mathrm{Nb}-\mathrm{Zr}$.

III. Transformation des alliages. - La mise en forme des alliages de métaux réfractaires nécessite en général une température élevée. Les techniques habituellement employées sont le forgeage et le laminage précédés éventuellement d'une extrusion. La facilité de transformation des alliages est liée aux teneurs en éléments d'alliages et en impuretés non métalliques: la température de transition ductilefragile est très sensible à la pureté du métal [4] [5].

L'aptitude à la mise en forme des alliages de niobium est généralement bonne comparée à celle des alliages des autres métaux réfractaires, Mo et $\mathrm{W}$; seuls les alliages de $\mathrm{Nb}$ à haute résistance mécanique nécessitent une extrusion préliminaire.

D'après Begley et al. [4] [6] les alliages Nb-Hf, $\mathrm{Nb}-\mathrm{V}, \mathrm{Nb}-\mathrm{Mo}$ et $\mathrm{Nb}-\mathrm{W}$ ont une bonne aptitude au forgeage et au laminage à chaud $\left(1200-1300^{\circ} \mathrm{C}\right)$ pour des teneurs $\leqslant 20 \%$. Les alliages $\mathrm{Nb}-\mathrm{Cr}$ et $\mathrm{Nb}-\mathrm{Zr}$ sont les plus difficilement transformables; certains alliages à faible teneur en zirconium sont toutefois laminables à froid jusqu'à $75 \%$ de réduction. Les meilleurs résultats sont obtenus avec $\mathrm{Nb}-\mathrm{Ti}$ et $\mathrm{Nb}-\mathrm{Y}$. Sheely [7] a transformé des alliages de niobium à $5 \%$ at. de $\mathrm{Ti}, \mathrm{Zr}$ et $\mathrm{V}$ à $1200^{\circ} \mathrm{C}$. Après une réduction de $50 \%$ par forgeage, les alliages subissent un recuit de $1 \mathrm{~h}$ à $1400{ }^{\circ} \mathrm{C}$ et sont laminés à froid jusqu'à $85 \%$ de réduction. Les températures atteintes au cours de ces opérations nécessitent l'emploi d'un revêtement protecteur contre l'oxydation. Les techniques généralement employées consistent à gainer les produits à transformer par de l'acier ou du molybdène ; certains. comme Sheely [7] utilisent une couche de verre visqueuse. Ces procédés sont peu commodes à mettre en œuvre, longs et coûteux.

De Mastry et Foster [8] ont réussi à transformer des alliages $\mathrm{Nb}-\mathrm{Zr}, \mathrm{Nb}-\mathrm{Mo}, \mathrm{Nb}-\mathrm{Cr}$ et $\mathrm{Nb}-\mathrm{Ti}-\mathrm{Cr}$ et $\mathrm{Nb}-\mathrm{Ti}$ Mo à $540^{\circ} \mathrm{C}$. Le choix de cette température permet d'éviter le gainage. Après le succès de cette opération, ils ont tenté de transformer à froid les alliages $\mathrm{Nb}-3,3 \% \mathrm{Zr}, \mathrm{Nb}-20,5 \mathrm{Ti}-4,28 \mathrm{Cr}$ et $\mathrm{Nb}-4,5 \mathrm{Mo}$. $\mathrm{Si}$ les deux premiers supportent un taux de réduction de $90 \%$, l'alliage $\mathrm{Nb}-4,5$ Mo crique sévèrement et rapidement.

Il apparaît donc que l'obtention de tôles minces et de fils de faible diamètre nécessite, en général, soit des températures élevées soit des recuits intermédiaires lors d'une transformation à froid. Afin de limiter au maximum la contamination en cours de transformation nous avons cependant tenté, et réussi, la transformation intégrale à froid et sans aucun recuit intermédiaire de tous les alliages élaborés.

1. FABRICATION DE TÔLES. - Les lingots ont un diamètre de $56 \mathrm{~mm}$ environ et un poids approximatif de $4 \mathrm{~kg}$.

Le premier stade du forgeage consiste à mettre le métal sous la forme d'un larget ou plat de $20 \times 60 \mathrm{~mm}$ de section à l'aide d'un marteau pilon de forte puissance. Le métal est forgé entre tas plats à grands rayons. Après un léger dégourdissage du métal ainsi que des tas, le forgeage débute, en utilisant une faible énergie, par une mise approchée en octogone. Le forgeage est poursuivi avec une énergie croissante, la vitesse de frappe restant peu élevée. Le métal chauffe légèrement au cours de cette opération mais, en fin de forgeage, sa température est néanmoins nettement inférieure à $200{ }^{\circ} \mathrm{C}$.

A partir du plat de $20 \mathrm{~mm}$ le forgeage est poursuivi, à l'aide d'un pilon de puissance plus faible et à vitesse de frappe plus élevée, jusqu'à l'obtention d'un plat de $12 \times 65 \mathrm{~mm}$ environ. A ce stade l'écrouissage est de $70 \%$ et les plats ne présentent aucune fissure. Ces plats sont partagés en deux parties égales et la réduction de l'une des moitiés est poursuivie par laminage.

Cette opération s'effectue sans difficulté pour les alliages Nb-2 Zr, Nb-Mo et Nb-Ti jusqu'à l'obtention de tôles de $1 \mathrm{~mm}$ d'épaisseur. Par contre, l'alliage $\mathrm{Nb}-5,4 \mathrm{Zr}$ crique dès la première passe de laminage. Une fissure interne a été décelée dans la partie centrale du plat forgé de $12 \mathrm{~mm}$. Après élimination de cette zone, le laminage d'une partie saine conduit à nouveau et rapidement à une fissure.

Des essais d'adoucissement par des recuits à $950^{\circ}$ et $1200^{\circ} \mathrm{C}$, de laminage à chaud à $500^{\circ}$ et $800^{\circ} \mathrm{C}$ se 
sont révélés infructueux. Nous avons alors tenté de poursuivre le forgeage à froid au-delà de $12 \mathrm{~mm}$.

Contre toute attente, cette tentative a réussi : le plat de $5 \mathrm{~mm}$ obtenu ne présente pas de criques et le taux d'écrouissage est de $87 \%$. Ce plat se lamine sans difficulté jusqu'au stade de la tôle de $1 \mathrm{~mm}$. L'alliage $\mathrm{Nb}-3,8 \mathrm{Zr}$ a été transformé de la même façon : il se lamine sans difficulté à partir du plat forgé de $7,2 \mathrm{~mm}$ dont le taux d'écrouissage est de $80 \%$.

Les tôles obtenues sont poncées et décapées en fin de laminage par un mélange nitro-sulfo-fluorhydrique. Leur état de surface est excellent et elles ne présentent aucune crique de rive. Le taux de réduction final est de $97 \%$.

2. FABRICATION DE FILS. - Une partie du plat forgé de $20 \mathrm{~mm}$ est forgée en rond de $14 \mathrm{~mm}$. Le fil est obtenu par martelage à froid. Le martelage se déroule sans difficulté ; des fils de $0,3 \mathrm{~mm}$ de diamètre sont obtenus, sans recuit intermédiaire, pour toutes les nuances d'alliages élaborés. Le taux d'écrouissage final de ces fils est de 99,95\% environ.

Conclusion. - L'élaboration d'alliages binaires de niobium dans un état de grande pureté constituait l'objectif principal de ce travail. Cet objectif peut être considéré comme atteint.

L'analyse des lingots laisse cependant apparaître une légère contamination par rapport aux métaux de départ. La principale origine de celle-ci est la mise en copeaux du niobium et des éléments d'alliage. Dans le cas de l'alliage $\mathrm{Nb}-\mathrm{Ti}$ la technique de fusion est une cause supplémentaire de contamination.

Les compositions initialement prévues n'ont pas toujours été atteintes par suite d'une mauvaise estimation des pertes en élément d'alliage en cours de fusion et de la difficulté de préparer un barreau aggloméré homogène. Le bilan avant transformation s'établit, pour les cinq alliages, comme suit : la quantité initiale de niobium est de $5,5 \mathrm{~kg}$ par alliage; après écroutage et élimination des extrémités, le poids approximatif du lingot est de $4 \mathrm{~kg}$. Le degré de pureté est supérieur à 99,97\%.

L'alliage $\mathrm{Nb}-\mathrm{Ti}$ se transforme aisément. La transformation à froid des alliages $\mathrm{Nb}-\mathrm{Zr}$ et $\mathrm{Nb}-\mathrm{Mo}$ est possible à condition de briser, avant laminage, leur structure de fusion à gros grains par un corroyage suffisant. Cette possibilité de transformation à froid limite donc au maximum la contamination après fusion.

Bibliographie

[1] Joly (F.) et SAINT-CricQ (J.), Annales des Mines, X-XI, 39-53.

[2] Verot (J. L.), Thèses Ingénieur Docteur, Strasbourg, 1963.

[3] Gonzales (R.), Muratori (E.), Frere (P.), Durand (R.), Mémoires Scientifiques Rev. Metallurg., 1967, LXIV, 403-411.

[4] Begley (R. T.) et Platte (W. N.), WAde Technical Report 57-344, Part IV.
[5] Ingramm (A. G.), Holden (F. C.), Ogden (H. R.) et JAFFEE (R. I.), Trans. AIME, 1961, 221, 517.

[6] Begley (R. T.), Bechtold (J. H.), ( Nb, Ta, Mo et W ), Ed. A. G. Quarrel, Elsevier Publishing Company, 1961.

[7] Sheely (W. F.), J. Less Common Metals, 1962, 4, 291300.

[8] De Mastry (J. A.) et Foster (E. L.), Columbium Metallurgy (Metallurgical Society Conferences, 10) Interscience, New York, London, 1961, 75-83, 